\title{
Communication
}

Velpula Divya and M. V. Sangaranarayanan*

\section{Electrodeposition of Polymer Nanostructures using Three Diffuse Double Layers: Polymerization beyond the Liquid/Liquid Interfaces}

https://doi.org/10.1515/eetech-2018-0002

Received Apr 16, 2017; accepted Dec 25, 2017

\begin{abstract}
Nanostructured conducting polymers have received immense attention during the past few decades on account of their phenomenal usefulness in diverse contexts, while the interface between two immiscible liquids is of great interest in chemical and biological applications. Here we propose a novel Electrode $_{(\text {solid) }} /$ Electrolyte $_{(\text {aqueous })} /$ Electrolyte $_{(\text {organic) }}$ Interfacial assembly for the synthesis of polymeric nanostructures using a novel concept of three diffuse double layers. There exist remarkable differences between the morphologies of the polymers synthesized using the conventional electrode/electrolyte method and that of the new approach. In contrast to the commonly employed electrodeposition at liquid/liquid interfaces, these polymer modified electrodes can be directly employed in diverse applications such as sensors, supercapacitors etc.
\end{abstract}

Keywords: Electropolymerization, Polyaniline, Polypyrrole, Polyindole, Polythiophene, Surfactants, Electrode/Electrolyte/Electrolyte Interface, Electrical double layer

\section{Introduction}

The study of the interface between two immiscible liquids is of much interest in many areas of science and technology. The first experimental evidence for ion transport at the interface between phenol and water was provided by Nernst and Riesenfeld [1], while the fundamental analysis was further advanced by Koryta et al. [2] indicating the iso-

\footnotetext{
^Corresponding Author: M. V. Sangaranarayanan: Department of Chemistry, Indian Institute of Technology - Madras Chennai 600 036 India; Email: sangara@iitm.ac.in; Tel.: +91-044-22574209; Fax: +91-044-22570545

Velpula Divya: Department of Chemistry, Indian Institute of Technology - Madras Chennai 600036 India
}

morphism between the liquid/liquid interfaces and biological membranes. In the past few decades, novel synthetic protocols for nanomaterials have emerged at liquid/liquid interfaces [3, 4] apart from studies of charge transfer, photoelectric effects, electrodialysis, drug behavior, electrocatalysis [5, 6] and computer simulations [7]. In a complementary manner, diverse experimental techniques such as voltammetric (using four electrodes [8], thin film and three electrode approaches [9], three phase electrode assemblies [10], etc.) and characterization studies viz NMR spectroscopy [11], scanning electrochemical microscopy [12], $\mathrm{X}$-Ray and neutron scattering techniques [13], electron paramagnetic resonance spectroscopy [14] have been used for mechanistic analysis of the interfacial processes. The study of liquid/liquid interfaces is of paramount interest in biology [15], chemistry [16] and medicine [17], while the phase transfer studies between two immiscible liquids have great importance in fundamental understanding of interfacial phenomena and also in wide applications viz controlled encapsulation, delivery of substances etc [18].

Conducting polymers of micro- and nanometer size have been investigated on account of their potential as electronic and electrochromic devices [19], sensors [20] and light-emitting diodes [21]. Polyaniline (PANI)-based compounds are especially promising on account of their ease of synthesis, adjustable conductivity, processability and redox activity thus yielding various applications [22]. Apart from PANI, another conducting polymer of importance is polypyrrole (PPy) due to its large conductivity, satisfactory specific capacitance, thermal stability and impressive redox behaviour [23]. These conducting polymers with high surface area and porosity function effectively as the electrode materials for supercapacitors on account of their distinct conducting pathways, surface interactions and nanoscale dimensions [24]. In this context, polyindole (PIn) has not received much attention, due to slow polymerization kinetics despite its excellent environmental stability [25], conductivity [26] and high-redox activity [27]. Polythiophenes (PT) too possess large thermal stability as well as conductivity and can be employed in su- 
percapacitors [28], sensors [29], batteries [30] etc. The foregoing analysis indicates the wide range of applications of polymeric nano- and micro- structures. Hence it is of interest to investigate new methods for the electrochemical synthesis of polymers exploiting the full potentialities of $\mathrm{L} / \mathrm{L}$ interfaces in obtaining interesting morphologies vis a vis novel applications.

The experimental methodology proposed here is entirely new and different from any of the hitherto known studies at liquid/ liquid interfaces. It is customary to carry out deposition of species using heterogeneous electron transfer agents (HET) in four-electrode assemblies, thin film and three electrode approaches. On the other hand, the salient features of the present strategy are as follows: (i) no HET agents such as $\mathrm{M}^{n+} / \mathrm{M}^{(n-1)+}$ are employed here to induce the polymerization [31]; (ii) in contrast to the synthesis of polymer nanostructure using fourelectrode assembly where the working area constitutes the liquid/liquid interface and two reference as well as two counter electrodes are necessary [32], our experimental protocol involves a simple three-electrode assembly (iii) since the deposition occurs on the electrode surface, through the liquid/liquid interface here, direct applications of the modified electrode can be envisaged in contrast to the thin film electrode approach, wherein the deposited molecules are present on the electrode surface covered with a thin organic film [33] and (iv) in hithertoknown three-electrode polymerization methods, the electrodes are placed in the organic phase containing the monomer, while the volume ratio of the aqueous phase cannot exceed ca. $2 \%$, due to the solubility and diffusion limitations [34]; however, in the present strategy, since all the electrodes are placed in the aqueous phase and the monomer is present in the other phase, the compositions of the two phases can be varied to the desired extent. In contrast to the earlier methodologies [33, 34], the present approach demonstrates the occurrence of the monomer transport from the organic phase to the aqueous phase through the immiscible liquid/liquid interface mediated by a surfactant and bias electric field. This methodology can be considered as electrodeposition 'using' liquid/liquid interfaces rather than ' $a t$ ' liquid/liquid interfaces. The deposition of polyaniline, polypyrrole, polythiophene and polyindole nanostructures has been carried out so as to indicate the generality of the method.

\section{Materials and Methods}

\subsection{Chemicals}

Aniline (SRL Chemicals India), pyrrole (Spectrochem India), thiophene (Spectrochem India), were employed after suitable distillations. Indole monomer (Sigma Aldrich), tetrabutyl ammonium perchlorate (Fluka), lithium perchlorate (Alfa Aesar), tetradecylsulfate sodium salt (Sigma Aldrich), dichloromethane extra pure (Merck) were used as received. Triple distilled water was used in all studies.

\subsection{Electrodes}

The 304 Stainless Steel foils (working electrodes) were procured commercially with the area as $1 \mathrm{~cm}^{2}$ and thickness $1 \mathrm{~mm}$ while Pt wire was the counter electrode, $\mathrm{Ag} / \mathrm{AgCl}$ being the reference electrode (CH Instruments, USA). The composition of the stainless steel (SS) electrode was estimated as $\mathrm{Cr}$ (19.85\%), $\mathrm{Ni}$ (7.78\%) and $\mathrm{Fe}$ (72.38\%) using Energy Dispersive Spectroscopy (EDS). All the potentials are reported here with reference to the $\mathrm{Ag} / \mathrm{AgCl}$ electrode.

\subsection{Instrumentation}

The cyclic voltammograms were recorded using the $\mathrm{CH}$ Instruments Electrochemical work station, CH660D. The SEM images were captured using (FEI) HR-SEM Quanta FEG 200 at suitable voltages and magnifications. The FTIR spectroscopy was carried out with $\mathrm{KBr}$ pellets using Bruker Alpha (ATR-IR) spectrometer and the UV-Visible absorption spectra were obtained using JascoV-650 spectrophotometer. All the experiments were carried out at room temperature $\left(25 \pm 1^{\circ} \mathrm{C}\right)$.

\subsection{Methodology}

\subsubsection{Electrode $_{(\text {solid })} /$ Electrolyte $_{(\text {aqueous })}$ /Electrolyte $_{(\text {organic) }}$ Interface assembly}

The cell employed here is a simple $25 \mathrm{~mL}$ glass vessel wherein the organic phase consists of $0.1 \mathrm{M}$ tetrabutyl ammonium perchlorate (TBAP) in $10 \mathrm{~mL}$ of dichloromethane (DCM) to which $46 \mu \mathrm{L}$ of aniline is added; the aqueous phase contains $10 \mathrm{~mL}$ of $0.01 \mathrm{M}$ sodium tetradecylsulphate (STS) in $0.1 \mathrm{M} \mathrm{LiClO}_{4}$. The three electrodes are placed in the aqueous phase without contacting the organic phase. 


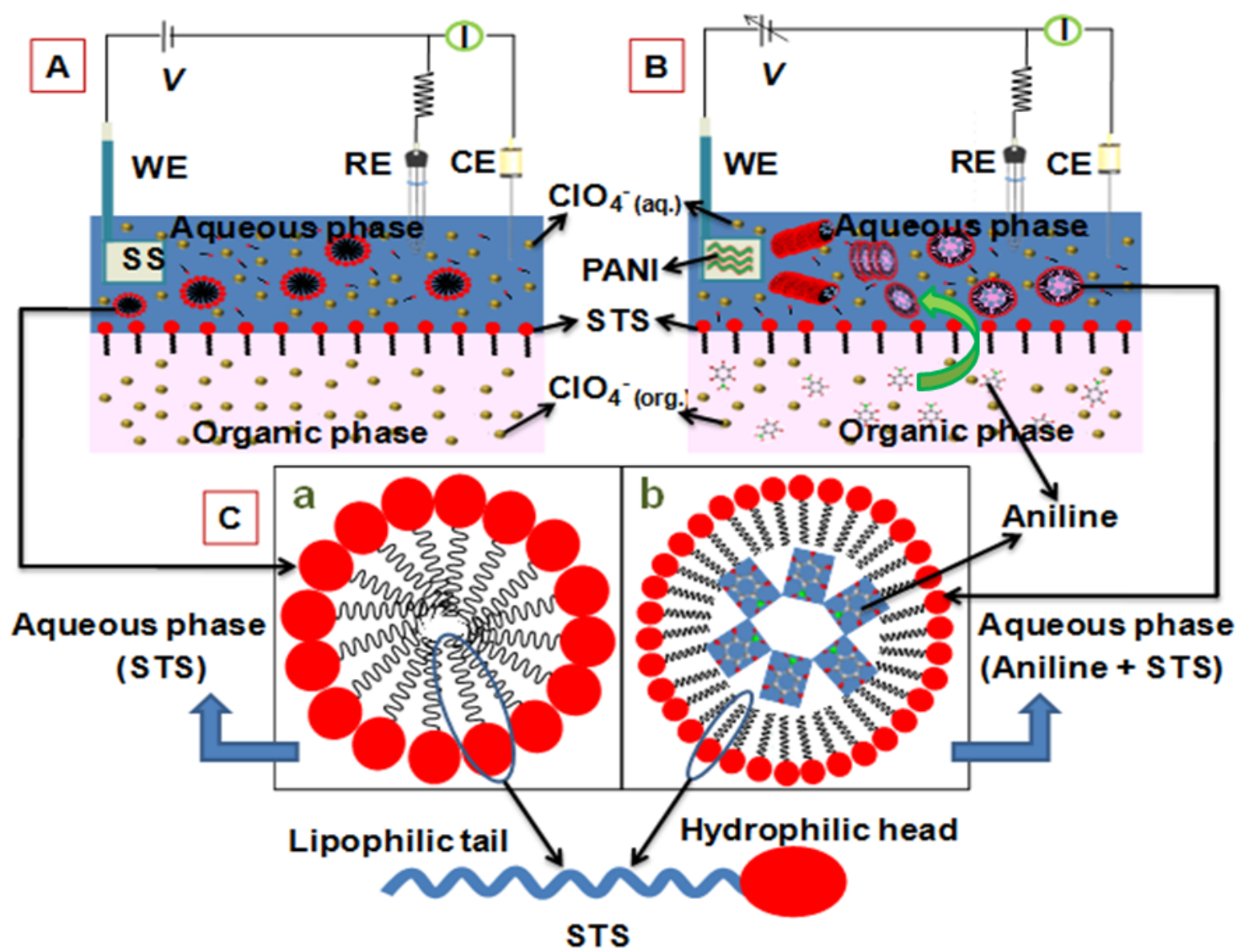

Figure 1: A schematic representation of the general EEEl assembly: (A) without any monomer and before applying the potential; (B) with monomer in the organic phase and after applying the potential. The curved green lines on the electrode in Figure $B$ indicate the formation of the polymer while the surrounding red colour denotes the surfactant. (C) The magnified view of the micelle forms: (a) surfactant alone and (b) the surfactant with the monomer in the aqueous phase. For specificity in the schematic representation, the monomer is chosen as aniline, the surfactant being sodium tetradecylsulfate.

\subsubsection{Electrode $_{(\text {solid) }} /$ Electrolyte $_{(\text {aqueous })}$ Interface assembly}

The same cell and electrodes are employed here too but the aqueous phase consists of $10 \mathrm{~mL}$ of $0.01 \mathrm{M} \mathrm{STS}$ and 0.1 $\mathrm{M} \mathrm{LiClO}_{4}$ and $46 \mu \mathrm{L}$ of aniline, while the organic phase is absent.

\section{Results and Discussion}

\subsection{Demonstration of EEEI methodology}

The formation of polyaniline on the SS electrode is demonstrated using water/dichloromethane interface assisted by the three diffuse double layers. The SS electrode is employed here due to its inexpensive nature and applicability in a supercapacitor [35] The existence of the two interfaces is of significance here viz between (i) the electrode/electrolyte $\left(\mathrm{SS} / \mathrm{LiClO}_{4(\text { aq. }}\right)$ and (ii) the two immiscible electrolyte solutions (Tetrabutyl ammonium perchlorate $(\mathrm{TBAP})_{(\text {org. }} /$ /ithium perchlorate $\left.\left(\mathrm{LiClO}_{4}\right)_{(\text {aq. }}\right)$. Hence this arrangement may be designated as the Electrode/Electrolyte (aq.) $_{\text {. }} /$ Electrolyte $_{(\text {org.) }}$ Interface system (EEEI system), it is depicted in Figure 1. Here, the sodium tetradecyl sulphate plays the role of a molecular pump in transporting monomers from one phase to another, analogous to that of ions or molecules through the biological membrane channels [36]. The driving force is the applied potential facilitating the transport of monomers towards the electrode/electrolyte interface at which the oxidation occurs. The formation of PANI is inferred from FTIR (Supporting Information Figure S1) spectral data. 

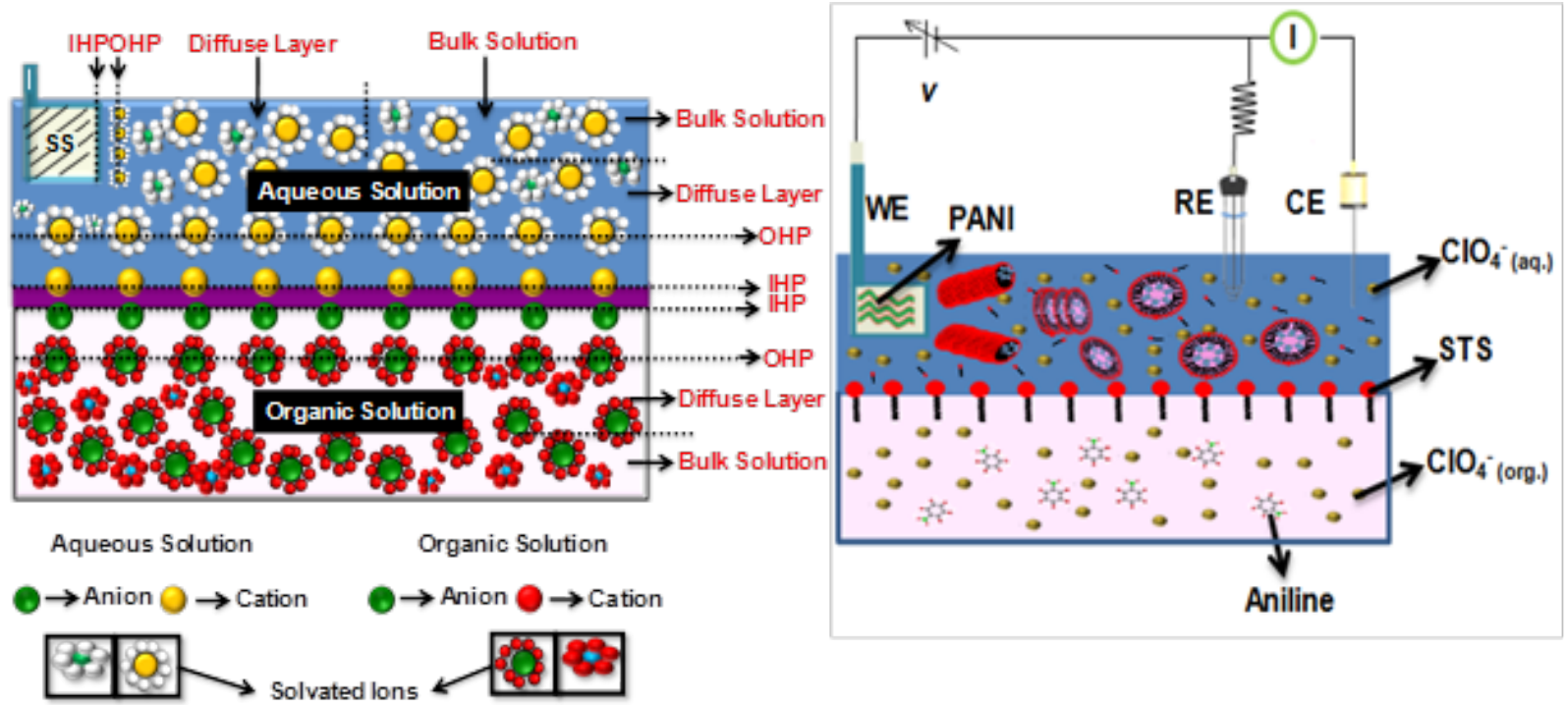

Scheme 1: Representation of three diffuse double layers viz. one at electrode/electrolyte and the other two at the aqueous electrolyte/organic electrolyte interfaces. IHP and OHP denote the inner Helmholtz plane and outer Helmholtz plane respectively.

\subsection{Visualization of three diffuse double layers}

The electrical double layer at electrode/electrolyte interfaces continues to be of interest in biological [37] and physical sciences [38]. The study of the electrical double layer is required in electrode kinetics, bacterial adhesion processes and non-viral gene therapy [39]. The electrode/electrolyte interface has one diffuse double layer [40] while the liquid/liquid interface possesses a compact layer surrounded by two diffuse double layers [41]. This visualization enables the comprehension of the experimental arrangement for the deposition of nanoparticles beyond liquid/liquid interfaces (Scheme 1).

\subsection{Potentiodynamic method for the electrodeposition of polyaniline using EEEl system}

Among various polymerization techniques, cyclic voltammetric method is preferable in view of the flexibility offered by the scan rate with a wide choice of the number of cycles. The schematic representation of the EEEI system is shown in Figure 2A. The appearance of the green colour on the working electrode and the cyclic voltammetric peaks (Figure 2B) and FTIR spectra (Figure S1, Supporting Information) are indicative of the polyaniline formation that has resulted due to the transfer of aniline from the organic phase to the aqueous phase. The cyclic voltammograms were obtained at the scan rate of $50 \mathrm{mV} \cdot \mathrm{s}^{-1}$ in the potential window of $-1.0 \mathrm{~V}$ to $1.5 \mathrm{~V}$, with the number of cycles being kept at 50 . The formation of polyaniline nanofibrous structures on the SS electrode is inferred from the SEM image of Figure 2C.

Interestingly, the nature and location of the voltammetric peaks are altered depending upon the parameters chosen implying the remarkable role of the chemical constituents for the electrodeposition of PANI nanostructures (Figure 3). Surprisingly, (i) even in the absence of the surfactant, PANI nanospheres are formed but to a lower extent indicating that STS enables a facile polymerization, (ii) the PANI nanofibers are formed in both cases with different orientations and aggregations indicating the role of the aqueous and organic supporting electrolytes $\left(\mathrm{LiClO}_{4}\right.$ and TBAP) on the morphologies and (iii) the well aligned nanospheres are noticed (in the presence of the surfactant) when the monomer is located in the aqueous phase. At this point, it is difficult to predict the exact reason for the self assembly of PANI spheres but the difference in morphologies here may be due to the partial diffusion of aniline from the aqueous to the organic phase. From the SEM images, it is inferred that the morphological changes may be implicitly attributed to the Galvani potential difference at the liquid/liquid interface and to the potential gradient at the interface between electrode/electrolyte rather than the surfactant. 


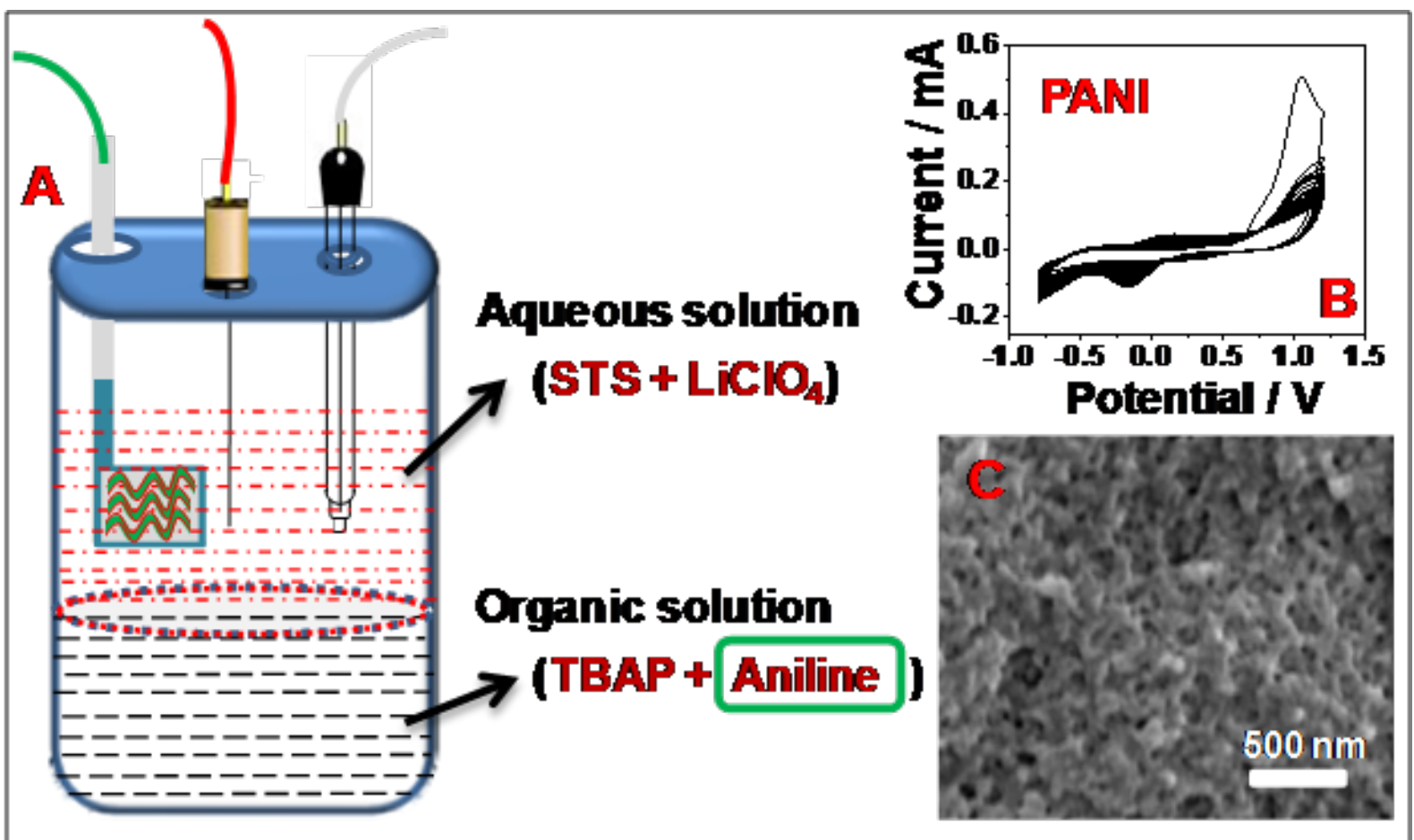

Figure 2: (A) Schematic depiction of the EEEI cell; (B) cyclic voltammogram for the polymerization of aniline and (C) a typical SEM image of PANI. Scan rate: $50 \mathrm{mV} \cdot \mathrm{s}^{-1}$, number of cycles: 50 .

\subsection{Uniqueness of EEEI strategy over conventional electrode/electrolyte interfacial system}

In order to further comprehend the uniqueness of the present strategy 'beyond' the $\mathrm{L} / \mathrm{L}$ interface, polyaniline has also been deposited using the conventional electrode/electrolyte interfacial system. Here, threedimensional PANI microspheres are observed and the morphologies are not uniform in contrast to the EEEI approach (Figure 4) indicating the novelty of our strategy for the synthesis of uniform PANI nanostructures in a simple manner.

\subsection{EEEl system for other polymers}

For validating this methodology, the polymerization of pyrrole, thiophene and indole was also carried out. The cyclic voltammograms and FTIR spectra imply the formation of polypyrrole, polythiophene and polyindole (Figure S5, Supporting Information). The voltammograms also demonstrate that the formation of polymers assisted by the three diffuse double layers is a general feature and not specific to PANI alone. Polypyrrole exhibits circular platelike morphologies with different orientations along with nanofibers on it. On the other hand, nanospheres are observed in the case of polythiophene and polyindole.

\subsection{Monomer transport studies of aniline, pyrrole, indole and thiophene}

At a preliminary stage, it can be surmised that the transport of monomers from the organic to the aqueous phase is caused by the applied potential which initiates the electropolymerization on the working electrode. In order to comprehend the transport of aniline, pyrrole, thiophene and indole from the organic phase to the aqueous phase, the UV-Visible spectra (Figure 5) were recorded for the aqueous solution, after electrodeposition (E) and without applying any potential (C), keeping identical time durations. ${ }^{1}$ Surprisingly, the transport of aniline, pyrrole, thiophene and indole was observed even in the absence of

1 The calculation of time duration pertaining to the polymerization is an involved exercise since the degree of polymerization increases as the potential is made more positive. An approximate value is obtained from the relation $|\Delta E|=$ scan rate $\mathrm{x}$ time where $\Delta E$ denotes the potential window. This value upon multiplication by the number of cycles may be considered as the total time of polymerization. However, it is essential to recognize that the applied potential may not al- 

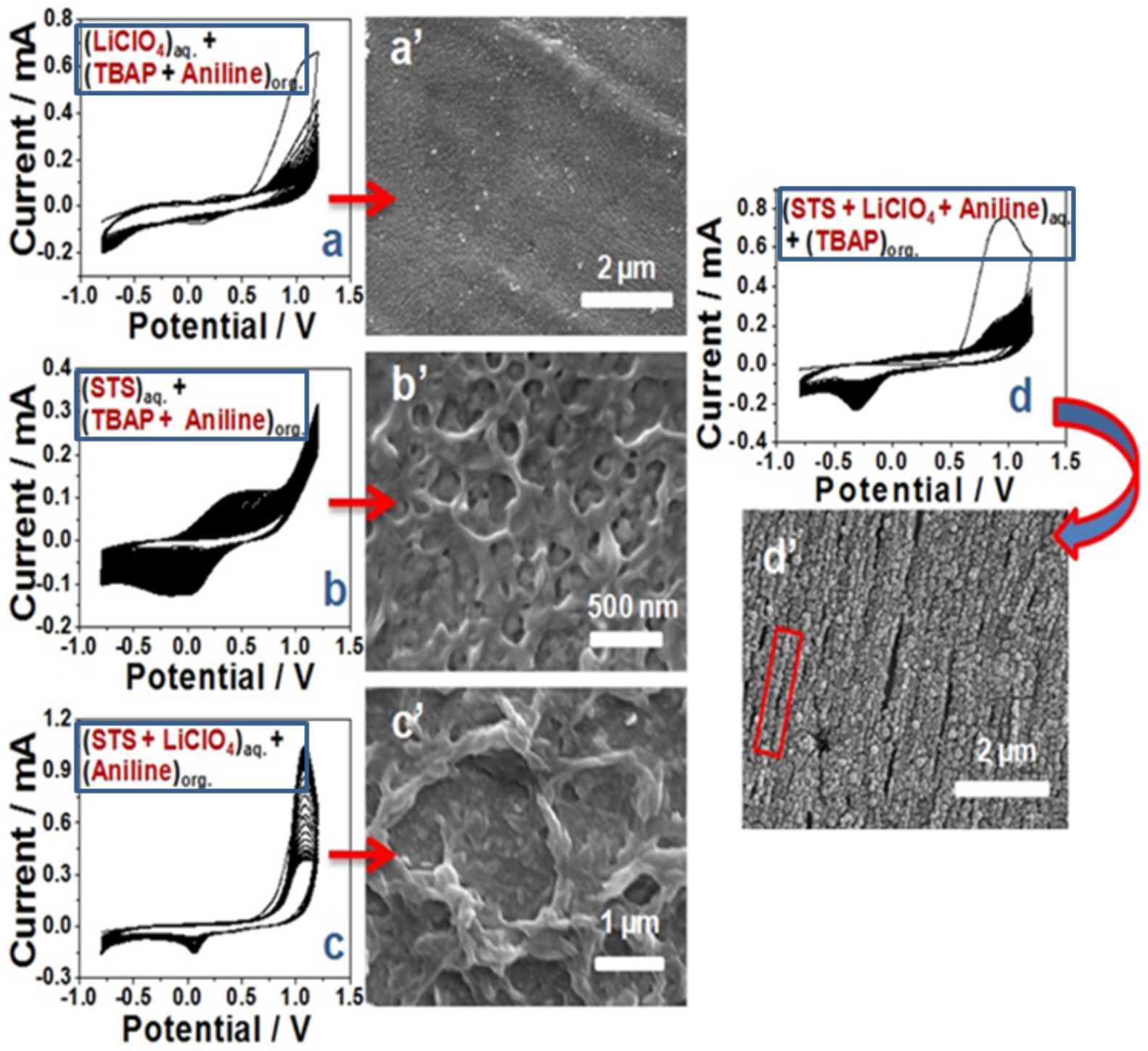

Figure 3: Polymerization of aniline using EEEl methodology: $a, b, c$ and d - cyclic voltammograms and (a', b, c' and d') - the corresponding scanning electron micrographs. The inset indicates the various chemical constituents in each phase. Other conditions as in Figure 2.

the applied potential. In hindsight, this may be attributed to the presence of the concentration gradient associated with the thermodynamic distribution laws at the interface. However, the precise quantification of the electrical and chemical potential gradients requires further investigation.

The transport of aniline occurs in the absence of applied potential due to its solubility in water and STS also promotes the transport form the organic phase thus forming stable micelles in the aqueous phase. The appear-

ways be sufficiently positive to facilitate electropolymerization. This aspect requires further investigation. ance of an additional peak at $560 \mathrm{~nm}$ in the UV-Vis spectra (Figure 5a) indicates the presence of PANI [38] and is interpreted as follows: during electrodeposition, PANI molecules near the electrode surface may diffuse away from the electrode and reach the bulk solution [39]. As pyrrole is partially hydrophilic, the gradient in the chemical potentials brings the molecules from the organic to the aqueous phase in a facile manner, thus the absorbance in UV-Vis spectra are analogous in both cases (C) and (E) mentioned above (Figure 5b). In the case of indole, the absorbance (below $300 \mathrm{~nm}$ ) increases with the applied potential due to its hydrophobic nature (Figure $5 \mathrm{c}$ ). The absorbance increases six times in the presence of the applied potential for thiophene which implies the crucial role in 

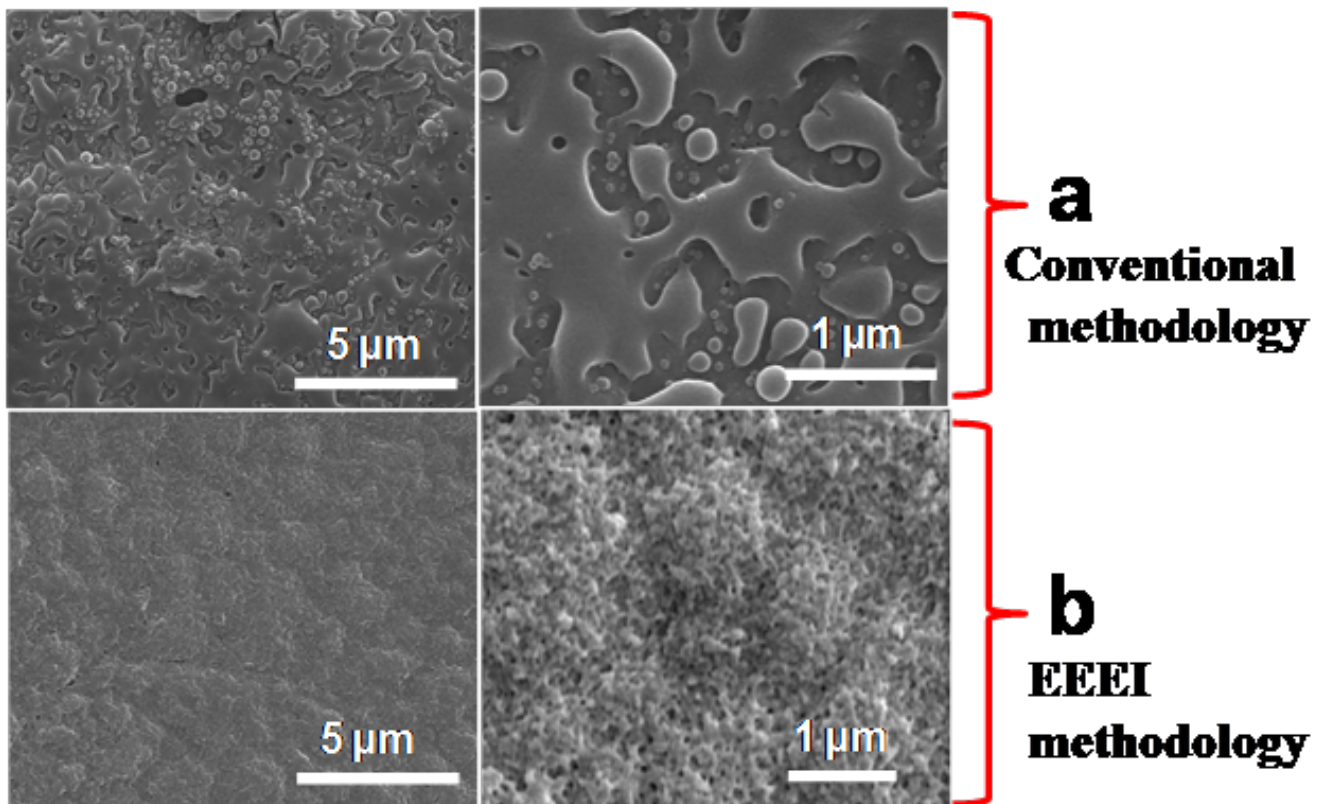

Figure 4: SEM image of polyaniline on the SS working electrode synthesized (a) at electrode/electrolyte interface and (b) using EEEI methodology; scan rate $=50 \mathrm{mVs}^{-1}$ and number of cycles $=50$.
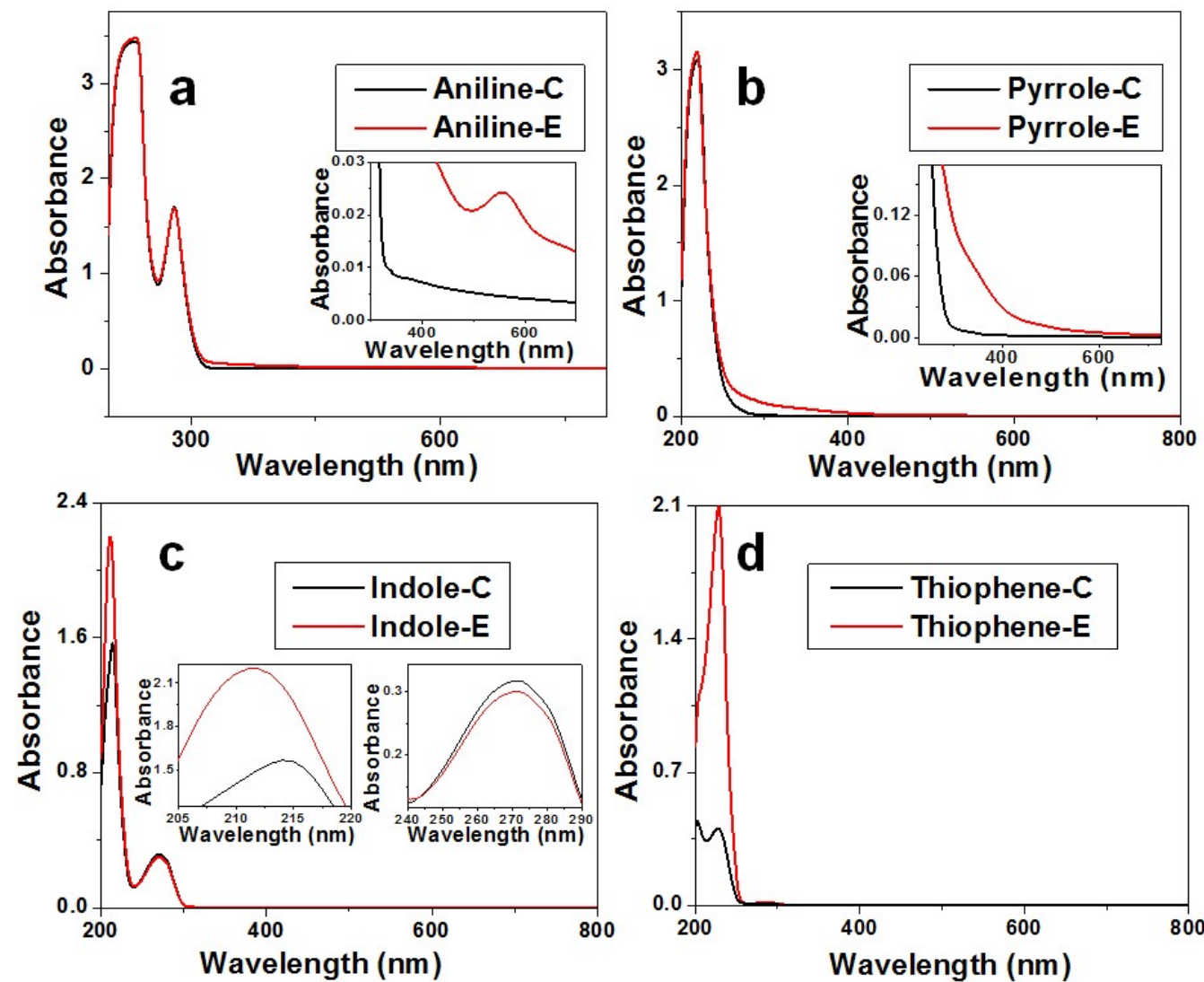

Figure 5: UV-Visible spectra demonstrating the molecular transfer from the organic to the aqueous phase with and without applied potential (E and $C$ respectively) for (a) aniline; (b) pyrrole; (c) indole and (d) thiophene. The insets in $a, b$ and $c$ denote the magnified view of the spectra. 
transporting the hydrophobic monomers from the organic to the aqueous phase (Figure $5 \mathrm{~d}$ ).

Thus, from the UV-Vis spectra it is inferred that the transfer occurs essentially due to the surfactant, while potential also plays a role in the case of indole and thiophene monomers (Figure S7, Supporting Information).

It is of interest to enquire whether the present interfacial synthetic method has any unique features. In order to respond to this, we emphasize that the transport of monomers which occurs from the organic to the aqueous phase can be controlled by structure-directing agents i.e. surfactants, apart from different types of aqueous and organic phase electrolytes. This flexibility is capable of yielding various morphologies of polymers. Furthermore, as an illustration, the potentiodynamic method has been chosen here although other electrochemical techniques may also provide additional insights into the growth mechanism. The choice of the stainless steel electrode was deliberate since the PANI-covered SS electrodes have earlier been demonstrated as suitable pseudocapacitor electrodes. It is envisaged that the present EEEI method will throw more light on the influence of synthetic techniques on the magnitude of the specific capacitance of electrochemical supercapacitors.

\section{Conclusions}

The concept of EEEI in conjunction with the three diffuse double layers is introduced for the deposition of conducting polymer nanostructures. Since the polymer nanostructures are formed directly on the electrode, through the liquid/liquid interface, this approach is different from the hitherto known deposition methods 'at' immiscible liquid/liquid interfaces and can be used directly for diverse applications viz. catalysis, sensors and supercapacitors etc. In view of the existence of the three diffuse double layers, new theoretical analysis for comprehending the equilibrium properties and the molecular transport process becomes essential. Further, the influence of the Gibbs free energy of transfer of monomers from the organic to the aqueous phase with subsequent reaction at the electrode/electrolyte surfaces requires a complete thermodynamic systems analysis. This method also implies diverse potential applications on account of the electrode/electrolyte and liquid/liquid interfaces existing in a single experimental system.

Acknowledgement: The helpful comments of the reviewers are gratefully acknowledged. We thank Prof. Wolfgang
Schmickler for several valuable suggestions on the applicability of diffuse double layers regarding the electrochemical synthesis of conducting polymers. This work was supported by SERB (EMR/2016/000379), Government of India.

\section{References}

[1] Nernst W., Riesenfeld E.H., Ann. Phys., 1901, 8, 600-608

[2] Koryta J., Ruth W., Vanysek P., Hofmanova A., Anal. Lett., 1982, 15, 1685-1692

[3] Janchenova H., Stulık K., Marecek V., J. Electroanal. Chem., 2006, 591,41-45

[4] Platt M., Dryfe R.A.W.,Phys. Chem. Chem. Phys., 2005, 7, 18071814

[5] Schmickler W., Santos E., Interfacial Electrochemistry, Springer Verlag, Berlin, $2^{\text {nd }}$ edition, 2010

[6] Volkov A.G.,DeamerD.W.,Liquid-Liquid Interfaces: Theory and Methods, CRC Press, 1996

[7] Sieffert N, Wipff G., Chem. Eur. J., 2007, 13, 1978-1990

[8] Aslan E., Patir I.H., Ersoz M., Chem. Eur. J., 2015, 21, 4585-4589

[9] Zhang Y., Bu C., Wang Y., Wang Z., Lu X., Liu X., Electroanalysis, 2012, 24, $1817-1823$

[10] Zhu H., Gao L., Li M., Yin H.Y., Wang D.H., Electrochem. Commun., 2011, 13, 1479-1483

[11] Mantel C., Bayle P.A., Hediger S., Berthon C., Bardet M., Magn. Reson. Chem., 2010, 48, 600-606

[12] Lu X., Gu W., Sun R., Liu X., Electroanalysis, 2012, 24, 2341-2347

[13] Elsen A., Festersen S., Runge B., Koops C.T., Ocko B.M., Deutsch M., Seeck O.H., Murphy B.M., Magnussen O.M., Proc. Natl. Acad. Sci.,2013, 110, 6663-6668

[14] Holze R., Surface and Interface Analysis, An electrochemists Toolbox, Spinger-Verlag Berlin Heidelberg, 2009

[15] Poletti Papi M.A., Caetano F.R.,. Bergamini M.F., MarcolinoJunior L.H.., Mater. Sci. and Eng. C, 2017, 75, 88-94

[16] Chen L., Huang L., Zhu J., Chem. Commun., 2014, 50, 1594415947

[17] VolkovA.G., Ed., Liquid Interfaces in Chemical, Biological and Pharmaceutical Applications, Marcel Dekker, New York, USA, 2001

[18] Bao C., Horton J.M., Bai Z., Li D., Lodge T.P., Zhao B., J. Polym. Sci. Part B: Polym. Phys., 2014, 52,1600-1619

[19] Gunbas G., Toppare L.,Chem. Commun., 2012, 48, 1083-1101

[20] Anzenbacher P., Palacios M.A., Nat. chem., 2009, 1, 80-86

[21] Berggren M., Inganäs O., Gustafsson G., Rasmusson J., Andersson M.R., Hjertberg T., Wennerström O., Nature, 1994, 372, 444446.

[22] Kuila B.K., Nandan B., Böhme M., Janke A., Stamm M., Chem. Commun., 2009, 5749-5751

[23] Dubal D.P., Lee S.H., Kim J.G., Kim W.B., Lokhande C.D., J. Mater. Chem., 2012, 22, 3044-3052

[24] Attarzadeh N., Raeissi K., Golozar M.A., Prog. Org. Coat., 2008, $63,167-174$

[25] Wadatkar N.S,. Waghuley S.A., Egypt. J. Basic appl. sci., 2015, 2, 19-24

[26] Koiry S.P., Saxena V., Sutar D., Bhattacharya S., Aswal D.K., Gupta S.K., Yakhmi J.V., J. Appl. Polym. Sci., 2007, 103, 595-599

[27] Cai Z., Yang G., Synth. Met., 2010, 160, 1902-1905 
[28] Gnanakan S.R.P., Rajasekhar M., Subramania A., Int. J. Electrochem. Sci., 2009, 4, 1289-1301

[29] Li C., Shi G., ACS Appl. Mater. Interfaces 2013, 5, 4503-4510.

[30] Aradilla D., Estrany F., Casellas F., Iribarren J.I., Alemán C., Org. Electronics 2014, 15, 40-46.

[31] Ding Z., Quinn B.M., Bard A.J., J. Phys. Chem. B 2001, 105, $6367-$ 6374.

[32] Samec, Z., Marecek V., Koryta J., Khali M.W., J. Electroanal. Chem. 1977, 83, 393-397.

[33] Francois Quentel F.,MirceskiV., L'HerM ., Anal Chem 2005,77,1940-1949.

[34] Liu X.H., Yang J., Zuo G.F., Zhang K., Dong C.W., Lu X.Q., J. Phys. Chem. C 2008,112,148 -152

[35] Girija T.C., Sangaranarayanan M.V., Synthetic Metals 2006,156,244-250.
[36] Alberts B., Johnson A., Lewis J., Raff M., Roberts K., Walter P., Molecular Biology of the Cell, $5^{\text {th }}$ edition, Garland Science, New York, 2007

[37] Blank M., Electrical Double Layers in Biology, Plenum press, New York, 1986

[38] Bohinc K., Iglic V.K., Iglic A., Electrochim. Acta, 2001, 46, 30333040

[39] Smith J., Zhang Y., Niven R., Adv. Drug Delivery Rev., 1997, 26, 135-150.

[40] Park J.Y., Park S.M., Sensors, 2009, 9, 9513-9532

[41] Harinipriya S., Sangaranarayanan M.V., J. Phys. Chem. B, 2004, $108,1660-1666$

[42] Moulton, S.E., Innis P.C., Kane-Maguire L.A.P., Ngamna O., Wallace G.G., Curr. Appl. Phys., 2004, 4, 402-406.

[43] Sazou D., Kourouzidou M., Pavlidou E.,Electrochim. Acta, 2007, $52,4385-4397$ 


\section{Supplementary Information}

\section{FTIR Spectroscopy}

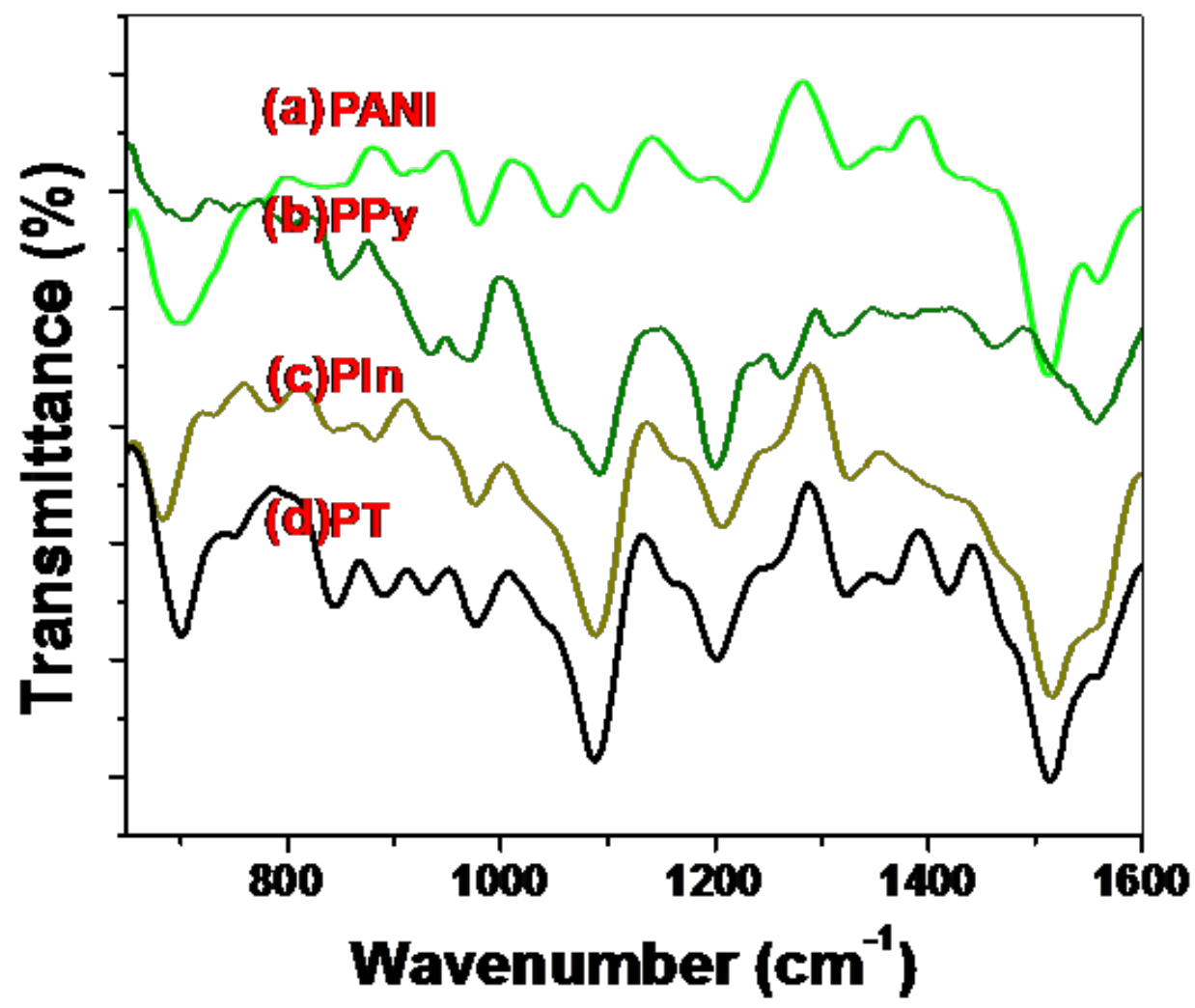

Figure S1: FTIR spectra of (a) polyaniline, (b) polypyrrole,(c) polyindole and (d) polythiophene

The characteristic FTIR peaks of PANI, PPy, PIn and PT:

\section{Polyaniline}

\begin{tabular}{cl} 
Frequency $/ \mathrm{cm}^{-1}$ & Assignment \\
\hline 833 & Aromatic C-H out of plane bending of the 1,4-disubstituted aromatic ring \\
778,1227 & C-N stretching vibration of aromatic amine out of plane deformation of C-H of 1,4 disubstituted \\
& rings \\
1101 & C=N stretching (-N=quinoid=N-) \\
1179 & Aromatic C-H bending in the plane for a 1,4 disubstituted aromatic ring \\
1322,1510 & C-N stretching (-N-benzenoid-N-) \\
1456 & $\mathrm{C}=\mathrm{C}$ stretching mode for the benzenoid rings \\
1558 & $\mathrm{C}=\mathrm{N}$ stretching modes for the quinoid rings \\
698 & $\mathrm{~S}-\mathrm{O}$ stretching \\
1054 & $\mathrm{~S}=\mathrm{O}$ stretching
\end{tabular}

The peaks are consistent with Li, N., Xiao, Y., Xu, C., Li, H., Yang, X., Int. J. Electrochem. Sci., 2013, 8, 1181-1188. 


\section{Polypyrrole}

\begin{tabular}{cl}
\hline Frequency $/ \mathrm{cm}^{-1}$ & Assignment \\
\hline 687 & C-C out of plane ring deformation or C-H rocking \\
804 & C-H out of plane ring deformation \\
934 & C-H out of plane deformation vibrations of the ring \\
970 & C-C out of plane ring deformation vibration \\
$1130-1000$ & C-H and N-H in plane deformation vibrations \\
$1400-1260$ & C-H or C-N in plane deformation modes \\
1459 & C-N stretching vibration in the ring \\
1555 & C-C stretching vibrations in pyrrole ring \\
\hline
\end{tabular}

The peaks are consistent with Omastova, M., Trchova, M., Kovarova, J., Stejskal, J., Synth. Met., 2003, $138,447-455$.

\section{Polyindole}

\begin{tabular}{cl}
\hline Frequency $\left(\mathrm{cm}^{-1}\right)$ & Assignment \\
\hline 730 & Out of plane deformation of $\mathrm{C}-\mathrm{H}$ bond in benzene ring \\
782 & Out of plane deformation of $\mathrm{C}-\mathrm{H}$ bond in pyrrole ring \\
973 & In plane deformation of $\mathrm{C}-\mathrm{H}$ bonds \\
$1130-1000$ & $\mathrm{C}-\mathrm{H}$ and $\mathrm{N}-\mathrm{H}$ plane of deformation \\
$1400-1260$ & C-N plane of deformation \\
1087,628 & Stretching of Cl-O bonds in perchlorate counter ions \\
1514 & Stretching of aromatic C-N bonds \\
\hline
\end{tabular}

The peaks are consistent with Talbi, H., Maarouf, E.B., Humbert, B., Umbert, M.A., Ehrhardt, J.J., Ghanbaja, J., Billaud, D., J. Phys. Chem. Solids, 1996, 57, I145-1151.

\section{Polythiophene}

\begin{tabular}{cl}
\hline Frequency $/ \mathrm{cm}^{-1}$ & Assignment \\
\hline 687 & C-C out of plane ring deformation \\
751 & C-S bending band \\
843 & C-S stretching \\
1021 & changes in the conjugated backbone due to the electron withdrawing \\
1420 & C=C asymmetric stretching of backbone \\
1323 & electron donating dopants on the polymer chain and the balancing counter ion $\mathrm{ClO}_{4}^{-}$ \\
1088 & vibration of counter ions in the polymer from the electrolyte solution $\mathrm{ClO}_{4}^{-}$
\end{tabular}

The peaks are consistent with Gnanakan, S.R.P., Rajasekhar, M., Subramania, A., Int. J. Electrochem. Sci., 2009, 4, 12891301. 


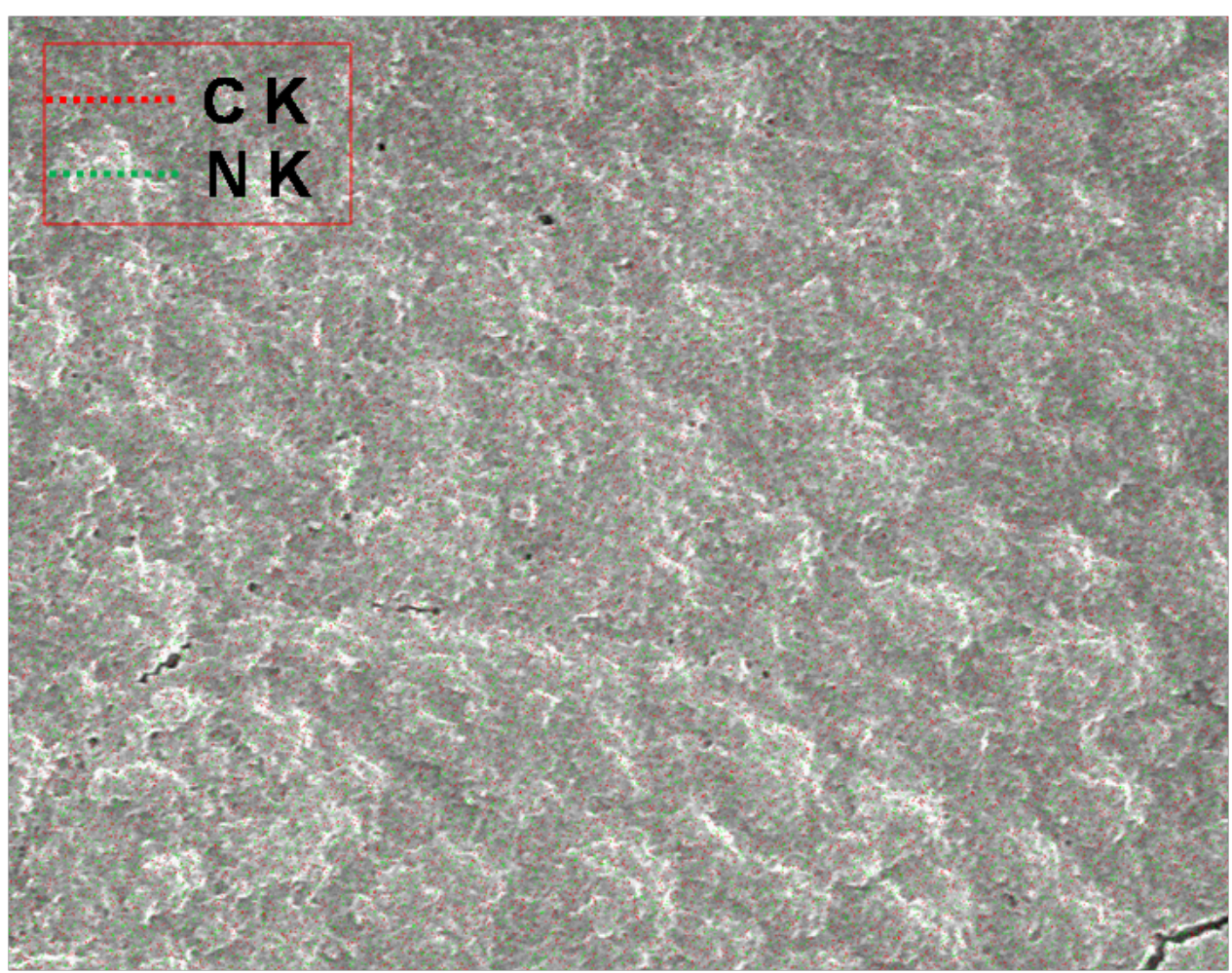

Figure S2: EDAX image of polyaniline depicting the locations of $\mathrm{C}$ and $\mathrm{N}$.

As anticipated, the scan rates and the number of cycles exert a marked influence on the morphologies. The formation of PANI nanofibers using EEEI system was also observed at the scan rates of $100 \mathrm{mVs}^{-1}$ and $500 \mathrm{mVs}^{-1}$ for 50 cycles, and at $500 \mathrm{mVs}^{-1}$ for 300 cycles. The increase in the scan rate leads to the aggregation of nanofibers on account of rapid diffusion of monomers towards the working electrode while the larger number of cycles results in the enhanced deposition on the electrode. The SEM images and the corresponding EDAX spectra are provided in Figures S3 and S4.
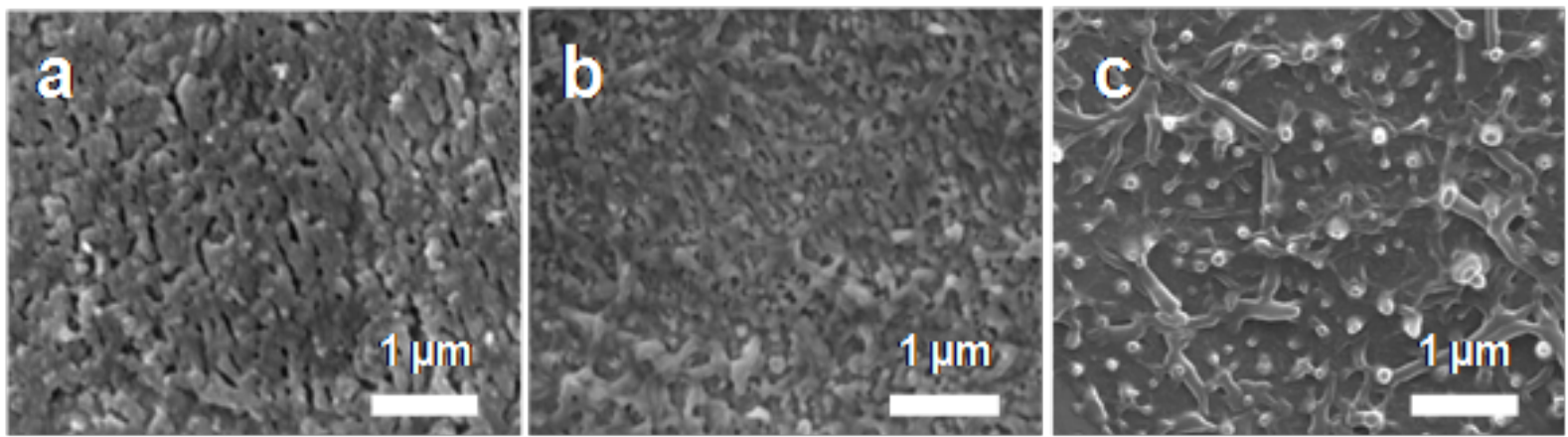

Figure S3: SEM images of polyaniline deposited on the the SS electrode using EEEl assembly. The aqueous phase (10 mL) contains 0.01 M STS in $0.1 \mathrm{M} \mathrm{LiClO}_{4}$ with the organic phase consisting of $0.1 \mathrm{M} \mathrm{TBAP,} 46 \mu \mathrm{L}$ of aniline, (a) scan rate $=100 \mathrm{mVs}^{-1}$; number of cycles $=50$, (b) scan rate $=500 \mathrm{mVs}^{-1}$; number of cycles $=50$ and (c) scan rate $=500 \mathrm{mVs}^{-1}$; number of cycles $=300$. The placement of the chemical constituents is as in Figure 2 of the main text. 


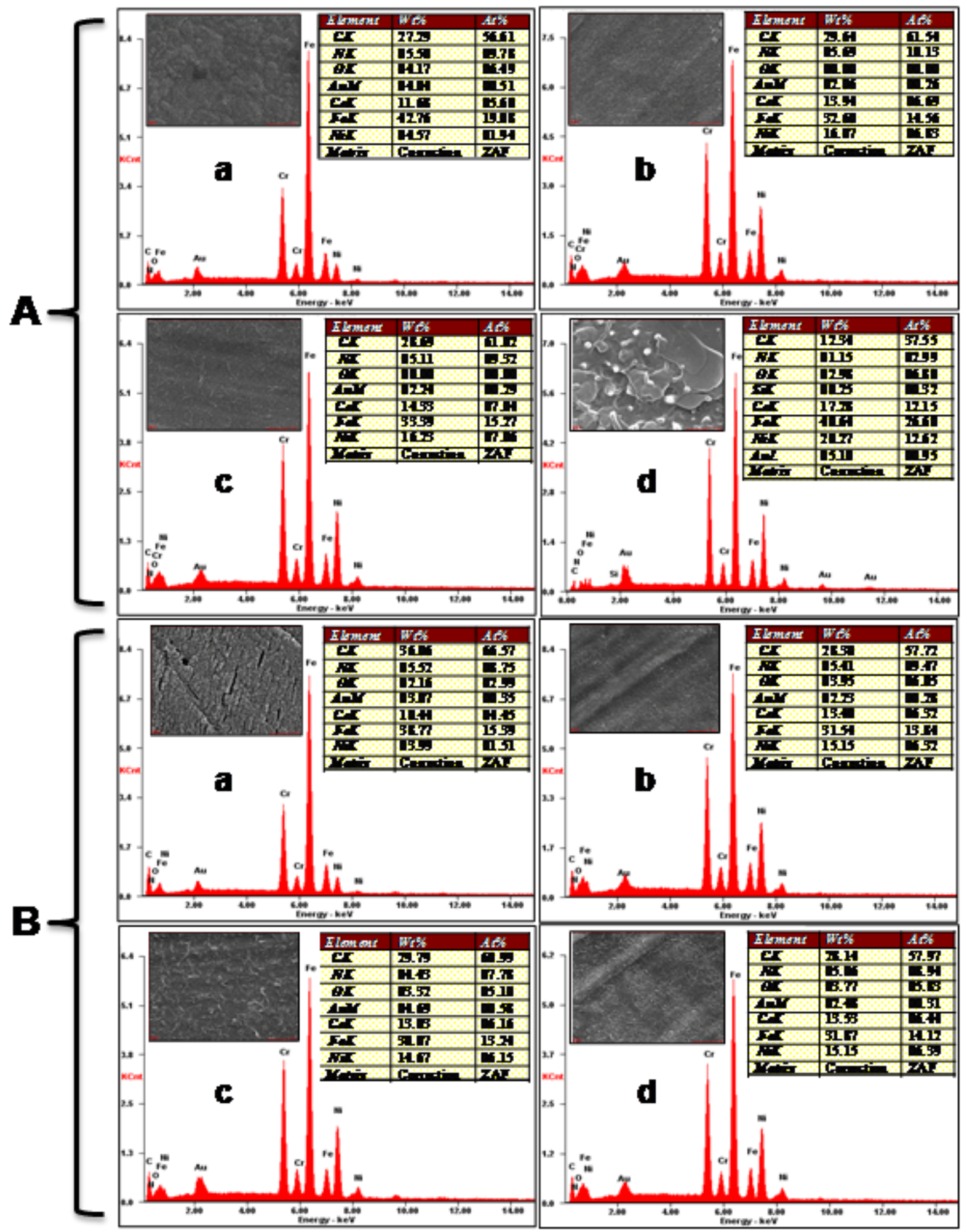

Figure S4: EDAX spectra of polyaniline formed on the SS electrode using EEEI strategy, with the aqueous phase consisting of $0.01 \mathrm{M}$ STS in $0.1 \mathrm{M} \mathrm{LiClO}_{4}$ while the organic phase contains $0.1 \mathrm{M}$ TBAP and $46 \mu \mathrm{L}$ of aniline in $10 \mathrm{~mL}$ of DCM, (A) for (a) scan rate $=50 \mathrm{mVs}^{-1}$; number of cycles $=50$; (b) scan rate $=100 \mathrm{mVs}^{-1}$; number of cycles $=50$; (c) scan rate $=500 \mathrm{mVs}^{-1}$; number of cycles $=50$ and (d) $\mathrm{scan}$ rate $=500$ $\mathrm{mVs}^{-1}$; number of cycles $=300$ and (B) scan rate $=50 \mathrm{mVs}^{-1}$; number of cycles $=50$ along with the conditions of (a) aniline in the aqueous phase, (b) without STS, (c) without TBAP and (d) without $\mathrm{LiClO}_{4}$. 


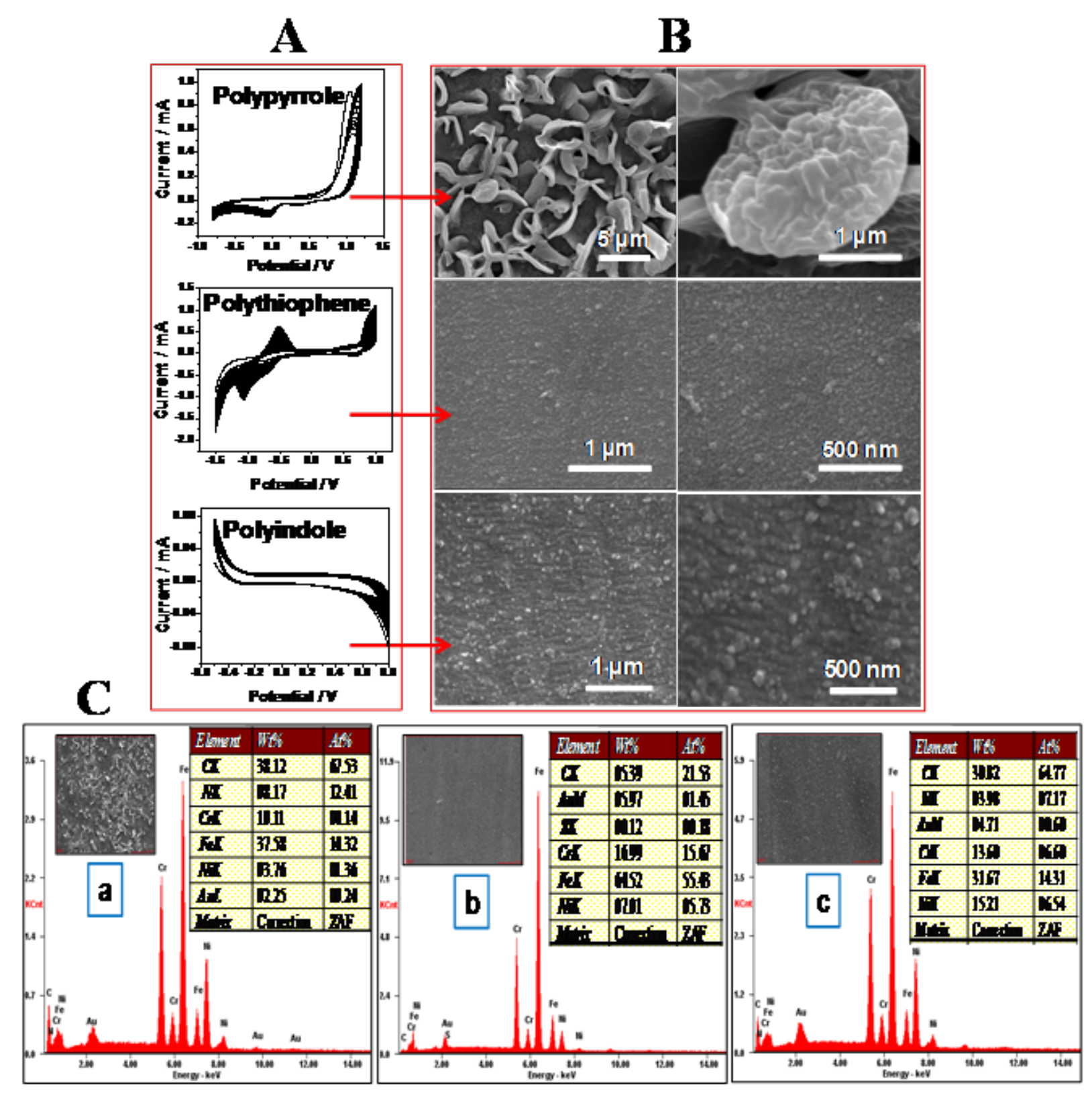

Figure S5: (A) Cyclic voltammograms depicting the polymerization of pyrrole, thiophene and indole using the EEEl system, (B) SEM images and $(C)$ the corresponding EDAX spectra (scan rate $=50 \mathrm{mVs}^{-1}$; number of cycles $=50$ ) while $16.6 \mu \mathrm{L}$ of pyrrole, $44 \mu \mathrm{L}$ of thiophene and $58.6 \mu \mathrm{L}$ of indole monomers were added to the organic phase for electropolymerization of respective monomers; other conditions are as in Figure 2 of the main text. 


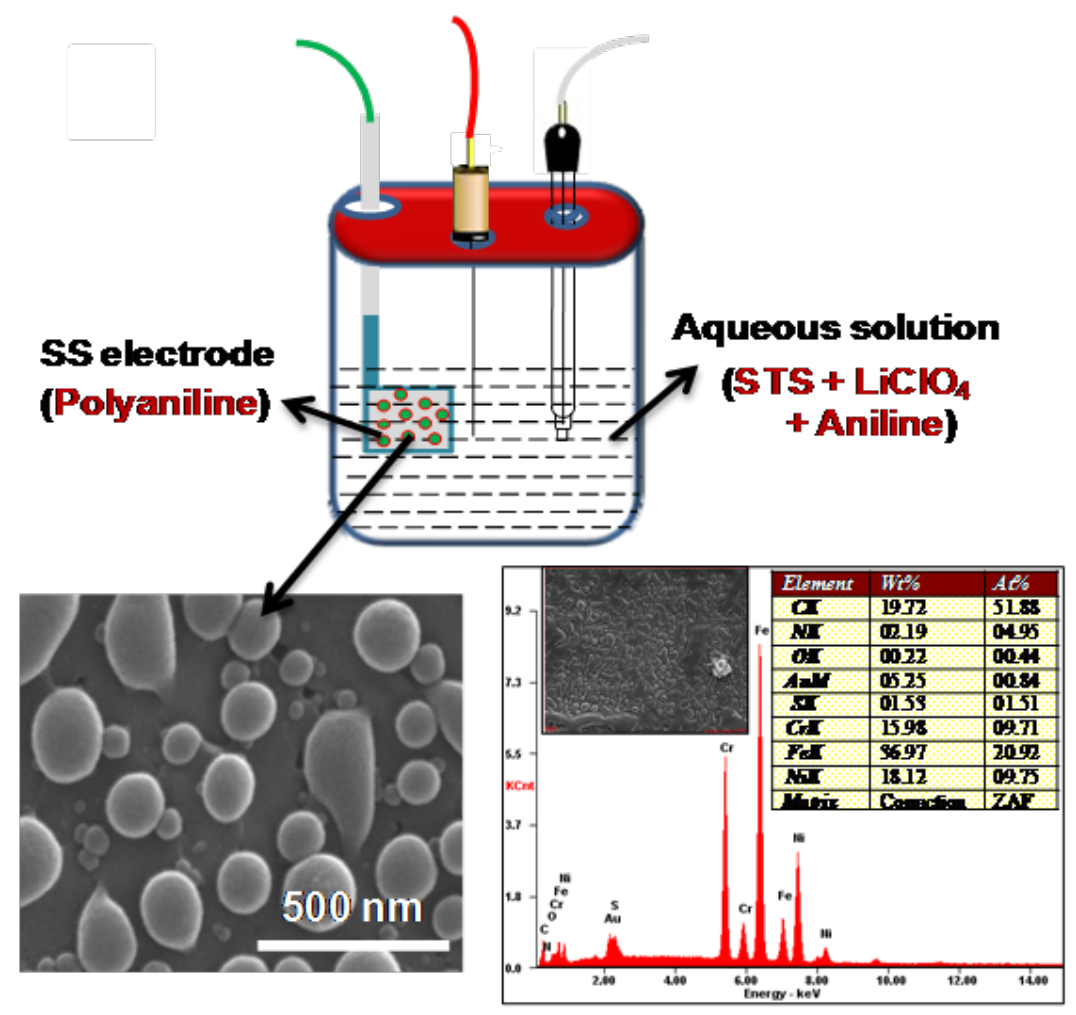

Figure S6: The schematic representation of the conventional method (electrode/electrolyte interface system) for the electropolymerization of aniline at the scan rate of $50 \mathrm{mVs}^{-1}$ for 50 cycles and the corresponding SEM image and EDAX spectra of polyaniline on the SS electrode. The aqueous phase contains $0.01 \mathrm{M}$ STS and $46 \mu \mathrm{L}$ of aniline in $10 \mathrm{~mL}$ of $0.1 \mathrm{M} \mathrm{LiClO}_{4}$.
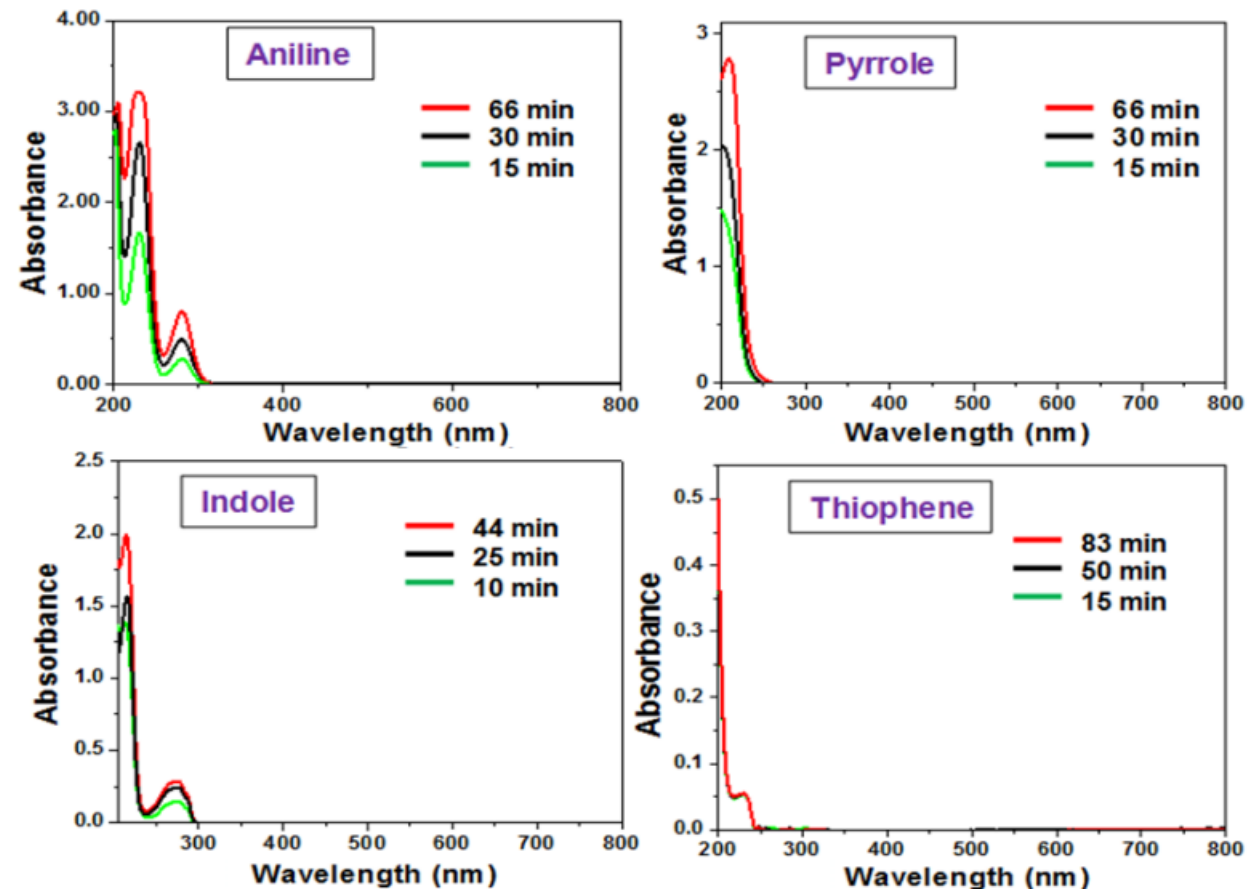

Figure S7: UV-Vis spectra demonstrating the transport of monomers from the organic to the aqueous phase at different time intervals. 